\title{
1E 1740.7-2942: Temporal and spectral evolution from INTEGRAL and RXTE observations *
}

\author{
M. Del Santo ${ }^{1}$, A. Bazzano ${ }^{1}$, A. A. Zdziarski², D. M. Smith ${ }^{3}$, N. Bezayiff ${ }^{3}$, R. Farinelli ${ }^{4}$, \\ G. De Cesare ${ }^{1}$, P. Ubertini ${ }^{1}$, A. J. Bird ${ }^{5}$, M. Cadolle Bel ${ }^{7}$, F. Capitanio ${ }^{1}$, A. Goldwurm ${ }^{7}$, A. Malizia ${ }^{6}$, \\ I. F. Mirabel ${ }^{7}$, L. Natalucci ${ }^{1}$, and C. Winkler ${ }^{8}$ \\ 1 Istituto di Astrofisica Spaziale e Fisica cosmica - CNR, via del Fosso del Cavaliere 100, 00133 Roma, Italy \\ e-mail: melania.delsanto@rm.iasf.cnr.it \\ 2 Nicolaus Copernicus Astronomical Center, Bartycka 18, 00-716 Warszawa, Poland \\ 3 Department of Physics, University of California, Santa Cruz, Santa Cruz, CA 95064, USA \\ 4 Dipartimento di Fisica, University of Ferrara, via del Paradiso 12, 44100 Ferrara, Italy \\ 5 School of Physics and Astronomy, University of Southampton, SO17 1BJ, UK \\ ${ }^{6}$ IASF/CNR, sezione di Bologna, via P. Gobetti 101, 40129 Bologna, Italy \\ 7 CEA Saclay, DSM/DAPNIA/SAp (CNRS FRE 2591), 91191 Gif sur Yvette Cedex, France \\ 8 Research and Scientific Support Department of ESA, ESTEC, Postbus 299, 2200 AG Noordwijk, The Netherlands
}

Received 23 September 2004 / Accepted 17 November 2004

\begin{abstract}
We present results of the monitoring of the black hole candidate 1E 1740.7-2942 with INTEGRAL, in combination with simultaneous observations by RXTE. We concentrate on broad-band spectra from INTEGRAL/IBIS and RXTE/PCA instruments. During our observations, the source spent most of its time in the canonical low/hard state with the measured flux variation within a factor of two. In 2003 September the flux started to decline and in 2004 February it was below the sensitivity level of the INTEGRAL and RXTE instruments. Notably, during the decline phase the spectrum changed, becoming soft and typical of black-hole binaries in the intermediate/soft state.
\end{abstract}

Key words. gamma rays: observations - radiation mechanisms: non-thermal - stars: individual: 1E 1740.7-2942 $\mathrm{X}$-rays: binaries - black hole physics

\section{Introduction}

The X-ray source 1E 1740.7-2942 was discovered in 1984 by the EINSTEIN observatory (Hertz \& Grindlay 1984) and its hard X-ray emitting nature was firstly reported by Skinner et al. (1987). Based on the spectral shape of its soft $\gamma$-ray emission and on the similarities with Cyg X-1, it was classified as a black hole candidate (BHC) and reported as the strongest persistent source in the Galactic Centre region (Sunyaev et al. 1991a).

After the discovery of a double-sided radio-emitting jet, 1E 1740.7-2942 was classified as a microquasar near the Galactic Centre by Mirabel et al. (1992). In 1991, it was also suggested as a possible positron-electron annihilation source due to a GRANAT/SIGMA observation of a high energy feature (Bouchet et al. 1991; Sunyaev et al. 1991b) in the spectrum, although its definitive confirmation has not been reported so far.

* Based on observations with INTEGRAL, an ESA project with instruments and science data centre funded by ESA member states (especially the PI countries: Denmark, France, Germany, Italy, Switzerland, Spain), Czech Republic and Poland, and with participation of Russia and the USA.
A simultaneous observation by CGRO/OSSE (Jung et al. 1995) has severely constrained the suggested feature intensity with an upper limit substantially below the flux measured by SIGMA (Cordier et al. 1993).

Most of the time, the source is observed in the typical BHC low/hard state, characterised in the X-ray domain by an absorbed power law with the photon index of $\Gamma \sim 1.4-1.5$ (Gallo \& Fender 2002) and a cutoff at high energies (Sidoli et al. 1999). The high hydrogen column density toward the source, recently estimated by Chandra as $N_{\mathrm{H}} \simeq$ $1.05 \times 10^{23} \mathrm{~cm}^{-2}$ (Gallo \& Fender 2002), does not permit any identification of its optical counterpart.

During RXTE observations of 1E 1740.7-2942, an interesting correlation between the time derivative of the flux and the hardness was discovered; no such correlation was found in Cyg X-1 (Smith et al. 2002a). A periodic modulation with amplitude $3-4 \%$ at $12.73 \pm 0.05$ days has been measured and interpreted as the orbital period, suggesting that the object could have a red-giant companion (Smith et al. 2002b). On the contrary, Martí et al. (2000) reported an upper limit for an IR counterpart with VLT that seems to exclude a red-giant companion. 
Table 1. INTEGRAL observations of 1E 1740.7-2942.

\begin{tabular}{ccccc}
\hline \hline Data Set & Observation Period & INTEGRAL JD $^{a}$ & Revolutions & Exposure (ks) \\
\hline GCDE $1 \backslash \mathrm{a}$ & $2003 / 02 / 28-2003 / 04 / 23$ & $1166.4-1205.6$ & $50-64$ & 690 \\
GCDE $1 \backslash \mathrm{b}$ & $2003 / 08 / 10-2003 / 10 / 14$ & $1317.7-1382.2$ & $100-122$ & 720 \\
XTE J1720-318 ToO & $2003 / 02 / 28-2003 / 03 / 02$ & $1154.2-1156.3$ & 46 & 168 \\
H1743-322 ToO & $2003 / 04 / 06-2003 / 04 / 22$ & $1191.6-1207.5$ & $58,61,63$ & 280 \\
GCDE 2 $\backslash \mathrm{a}$ & $2004 / 02 / 16-2004 / 04 / 20$ & $1507.7-1571.5$ & $164-185$ & 930 \\
\hline
\end{tabular}

${ }^{a}$ The INTEGRAL Julian Date (IJD) starts from 2000 January 1 and it corresponds to MJD = IJD + 51544.

Preliminary INTEGRAL results have been presented in Del Santo et al. (2004); they reported a flux variability of a factor of 2.5 and the absence of any correlation between photon index and $20-80 \mathrm{keV}$ flux during the low/hard state period. No spectral variability was presented, since the analysis was not completed at that time.

In the present work, we report on one year of almost simultaneous INTEGRAL and RXTE observations and on the first period of INTEGRAL second year observations. Details of the data set and scientific analysis are presented in Sect. 2. Temporal and spectral behaviour are reported in Sect. 3 and are discussed in Sect. 4.

\section{Observations and data analysis}

We analyse here INTEGRAL observations of 1E 1740.7-2942 performed in the framework of the Core Programme. This includes roughly 800 science windows ( $\mathrm{ScWs}$ ) collected during the first year of the Galactic Centre Deep Exposure (GCDE). The total exposure of $1.4 \mathrm{Ms}$ consists of two parts separated by $\sim 4$ months. The second year of the GCDE has not been completed yet; $930 \mathrm{ks}$ have been obtained so far and they are included in this work.

We have also added some pointings of the Targets of Opportunity (ToO) observations of XTE J1720-318 (Cadolle Bel et al. 2004) and H1743-322 (Parmar et al. 2003), corresponding to the new INTEGRAL source IGR J17464-3213 (Revnivtsev et al. 2003), which included 1E 1740.7-2942 in the field of view. In Table 1, we give the $\log$ of the observations. The INTEGRAL Core Programme pointings have a duration of $1800 \mathrm{~s}$ each for the GCDE and 2200 s each for the ToOs (see Winkler at al. 1999 for Core Programme details).

RXTE has also monitored 1E 1740.7-2942 since its first week of science operations in 1996. The monitoring began as monthly pointings of $\sim 1000-1500 \mathrm{~s}$, the frequency of which has increased over the years to about twice per week now. The RXTE observations simultaneous with those by INTEGRAL cover three different periods: 2003 March 13-April 26 with the total exposure of $\sim 19 \mathrm{ks} ; 2003$ August 12-24 with an exposure of $\sim 5 \mathrm{ks}$; and the third one, which consists of only two pointings at the beginning of 2003 October.

We use here data collected with the ISGRI (Lebrun et al. 2003) detector of the imager IBIS (Ubertini et al. 2003) on board INTEGRAL for the soft $\gamma$-ray range, and with the $R X T E / \mathrm{PCA}$ detector for the X-rays. The IBIS Partially Coded Field Of View (PCFOV) is $29^{\circ} \times 29^{\circ}$ at the zero response, but the full instrument sensitivity is achieved in the $9^{\circ} \times 9^{\circ}$ Fully Coded Field of View (FCFOV). For the temporal analysis, we took into account all the data, whereas for the spectral extraction, we selected only pointings with the source within the FCFOV, for which a reliable detector spectral response matrix is available.

Raw data corresponding to each pointing were preprocessed and organised in ScWs by the Integral Science Data Centre (Courvoisier et al. 2003) pipeline. The IBIS scientific data analysis software (Goldwurm et al. 2003) used is included in the INTEGRAL off-line analysis software (OSA) release 3.0. The lightcurves were extracted from the images in two energy bands: $30-50 \mathrm{keV}$ and $50-80 \mathrm{keV}$. The ISGRI spectra were extracted in 16 logarithmic bins and fitted using the response matrix corrected and tested by the IBIS team, and now delivered with the OSA 4.0 release.

The PCA data were analysed with FTOOLS v. 5.2 of the NASA High Energy Astrophysics Science Archive Research Center. Due to the crowded field near the Galactic Centre, the pointing axis was offset by $\gtrsim 0.5^{\circ}$ to avoid nearby sources. To eliminate the spectral contribution from the Galactic diffuse emission as much as possible, a background field was taken at a position symmetrical about the Galactic Centre from the source field. A map of the source and background fields with nearby sources is given in Main et al. (1999).

Spectral fitting was done with XSPEC v. 11.3.1 and single parameter uncertainties calculated at the $90 \%$ confidence level, i.e., for $\Delta \chi^{2}=2.71$. Systematic errors of $2 \%$ for the ISGRI spectra (which approximately corresponds to the current accuracy of the calibration) and 1\% for the PCA spectra have been added in quadrature.

\section{Results}

During the GCDE $1 \backslash \mathrm{a}$ (see Table 1), the 30-50 keV ISGRI count rate increased by $50 \%$. We measured the rate of $2.43 \pm$ $0.04 \mathrm{~s}^{-1}$ at the beginning of 2003 March and $3.66 \pm 0.33 \mathrm{~s}^{-1}$ after one month; these values correspond to $\sim 37$ and $\sim 56 \mathrm{mCrab}$, respectively. After four months, we observed a further increase up to $70 \mathrm{mCrab}$ in the rev. 103 (2003 August), in the same energy range. In 2003 September, the flux began to decline, and at the end of the GCDE 2003 it reached $25 \mathrm{mCrab}$.

In Fig. 1, we show the PCA lightcurve $(8-25 \mathrm{keV})$ together with two ISGRI lightcurves in the $30-50 \mathrm{keV}$ and $50-80 \mathrm{keV}$ energy ranges. Similar temporal behaviour appears in all three energy bands.

In 2004, when INTEGRAL pointed again at the Galactic Centre, the 1E 1740.7-2942 flux was dramatically lower. The source was detected at $\sim 10$ mCrab during rev. 164 and 


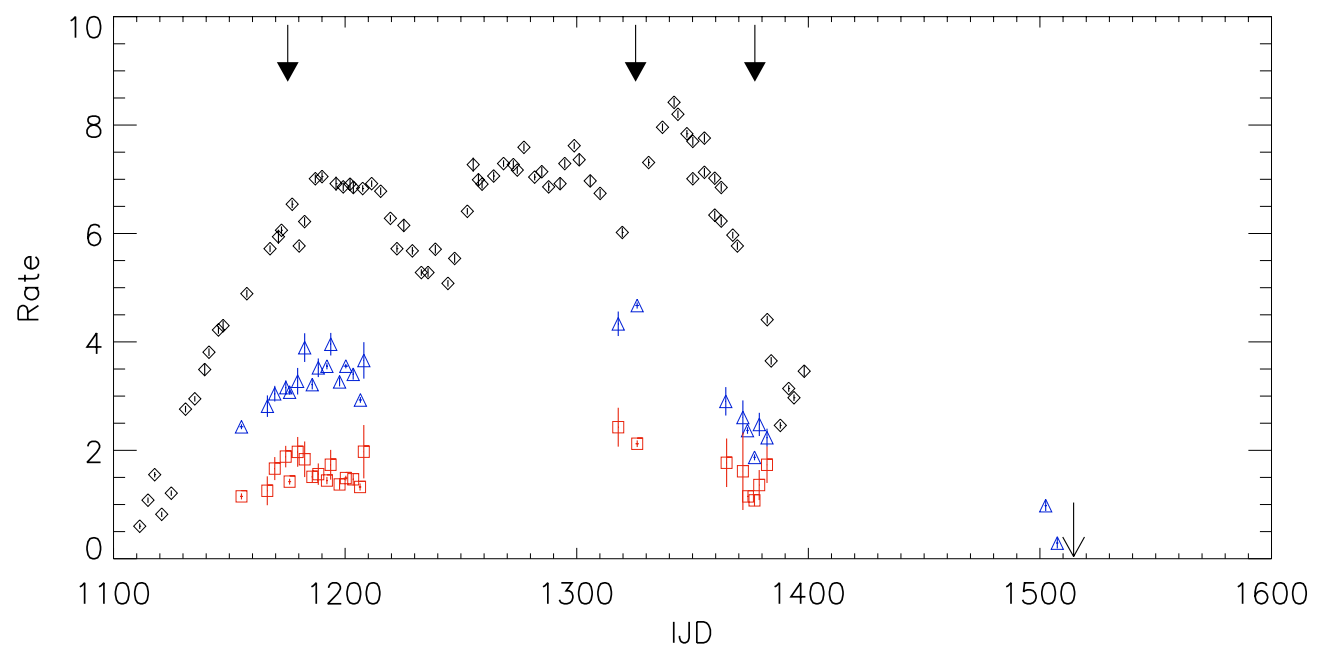

Fig. 1. Lightcurves from PCA (8-25 keV, black diamonds) and from IBIS/ISGRI in the two bands 30-50 keV (blue triangles) and 50-80 keV (red squares). Solid arrows indicate periods used for the spectral analysis. The differences in the sizes of the ISGRI error bars are due to the different exposure times. For clarity, the lightcurve for the $50-80 \mathrm{keV}$ band has been multiplied by 0.6 . Simple arrow indicates when the source was no longer visible by IBIS.

$\sim 3$ mCrab during rev. 165 (2004 February, IJD 1500). Soon after (simple arrow in Fig. 1) no detection was possible either with IBIS in $\gamma$-rays (also reported by Grebenev 2004) and with PCA in the X-ray domain (D. M. Smith, private communication). Such behaviour is not unusual, although rare; in spring 1991 the source flux went down to the GRANAT/SIGMA sensitivity level in the 40-150 keV range (Cordier et al. 1994).

To study the source spectral evolution, we have chosen three periods corresponding to different flux levels, namely rev. 53, 103 and 119-120, indicated by the solid arrows in Fig. 1. We initially fitted power law and an e-folded power law models. For rev. 53 and 103 we obtained the following best fit parameters with the $N_{\mathrm{H}}$ fixed at $1.05 \times 10^{23} \mathrm{~cm}^{-2}$ (using the model WABS in XSPEC): the photon index $\Gamma=1.59 \pm 0.03$ and the e-folding energy $E_{\mathrm{c}}=146_{-32}^{+53} \mathrm{keV}$ with $\chi_{v}^{2}=63 / 54$ for the first period, and $\Gamma=1.34 \pm 0.03, E_{\mathrm{c}}=90_{-13}^{+18} \mathrm{keV}$ with $\chi_{v}^{2}=63 / 54$ for the second one. The mean spectrum of the revolutions 119 and 120 was fitted by a simple power-law with $\Gamma=2.25 \pm 0.05$, which gives $\chi_{v}^{2}=82 / 51$.

Then, two thermal Comptonization models were used: COMPTT of Titarchuk (1994) and COMPPS $^{1}$ of Poutanen \& Svensson (1996).

COMPPS is a highly accurate iterative scattering Comptonization model, in which subsequent photon scatterings are directly followed (see, e.g., Zdziarski et al. 2000; Zdziarski et al. 2003): this model has been extensively tested against Monte Carlo results. On the other hand, COMPTT is based on an approximate solution of the kinetic equation with some relativistic corrections, and the resulting spectra are only approximate (see, e.g., discussion in the appendix of Zdziarski et al. 1996). Unlike COMPTT where the input spectrum of low-energy photons is a Wien law, COMPPS assumes for

\footnotetext{
1 Model available at ftp://ftp.astro.su.se/pub/juri/ XSPEC/COMPPS
}

the seed photons a simple blackbody or a multicolor disc blackbody spectrum.

We have allowed a free column density, but constrained it to $N_{\mathrm{H}} \geq 1 \times 10^{23} \mathrm{~cm}^{-2}$ (Gallo \& Fender 2002). With the former we have obtained a relatively low temperatures and high Thomson optical depths for the first two spectra, namely $k T_{\mathrm{e}}=26_{-3}^{+6} \mathrm{keV}$, $\tau=1.9_{-0.2}^{+0.3}, \chi_{v}^{2}=59 / 54$, and $k T_{\mathrm{e}}=20 \pm 2 \mathrm{keV}, \tau=2.9 \pm 0.1$, $\chi_{v}^{2}=55 / 53$, respectively. We have not been able to constrain the temperature for the third spectrum.

The fit results with the COMPPS are given in Table 2. We chose a geometry where Compton scattering is computed by the mean of the approximate treatment of radiative transfer using the escape probability for a sphere. The model also includes Compton reflection (Magdziarz \& Zdziarski 1995), which is found to be important for the rev. 103 (F-test probability of $\left.10^{-7}\right)$. We also fixed the ionization parameter at 0 as PCA has poor energy resolution. The three absorbed spectra are plotted in Fig. 2; in Fig. 3 the related residuals are shown. Figure 4 shows the intrinsic, unabsorbed spectra for rev. 53 and 119-120.

Our PCA data have been background-subtracted in a nonstandard way (Sect. 2). This procedure is supposed to remove, in particular, the Galactic ridge $\mathrm{Fe} \mathrm{K} \alpha$ emission. In our PCA data, we see no evidence of any Fe K line; however, this simply may be due to residual inaccuracies of the background subtraction.

Nearby sources are excluded by placing the centre of the $1^{\circ}$ field of view about $0.5^{\circ}$ away from 1E 1740.7-2942, and the strong Galactic diffuse background is subtracted with the help of pointings to an empty field symmetrically positioned across the GC. The spectra indicate an apparent state transition occurring from rev. 103 to $119-120$. The spectra for rev. 53 and 103 are consistent with those typical of black-hole binaries in the hard state whereas that for rev. 119-120 is more typical of the soft or intermediate state (Zdziarski \& Gierliński 2004). Surprisingly, the Compton reflection component appears the 
Table 2. The parameters of the PCA/ISGRI spectra fitted with the COMPPS model.

\begin{tabular}{lccccccc}
\hline \hline Rev. & $\begin{array}{c}N_{\mathrm{H}}{ }^{a} \\
10^{22} \mathrm{~cm}^{-2}\end{array}$ & $\begin{array}{c}k T_{\mathrm{bb}} \\
\mathrm{keV}\end{array}$ & $\begin{array}{c}k T_{\mathrm{e}} \\
\mathrm{keV}\end{array}$ & \multicolumn{6}{c}{$\Omega / 2 \pi$} & $\begin{array}{c}F_{\mathrm{bol}}{ }^{b} \\
\mathrm{erg} \mathrm{cm}^{-2} \mathrm{~s}^{-1}\end{array}$ & $\chi_{v}^{2}$ \\
\hline 53 & $12_{-2}^{+2}$ & $0.50_{-0.11}^{+0.06}$ & $110_{-30}^{+20}$ & $1.1_{-0.3}^{+0.6}$ & $0.3_{-0.2}^{+0.2}$ & $3.6 \times 10^{-9}$ & $70 / 51$ \\
103 & $11_{-1}^{+2}$ & $0.41_{-0.10}^{+0.09}$ & $150_{-50}^{+60}$ & $0.9_{-0.4}^{+0.4}$ & $0.9_{-0.2}^{+0.3}$ & $4.8 \times 10^{-9}$ & $50 / 51$ \\
$119-120$ & $10^{+1}$ & $0.68_{-0.05}^{+0.06}$ & $70_{-8}^{+11}$ & $1.0_{-0.2}^{+0.2}$ & $0.0^{+0.1}$ & $2.1 \times 10^{-9}$ & $44 / 47$ \\
\hline
\end{tabular}

a Constrained to $\geq 10^{23} \mathrm{~cm}^{-2}$.

$b$ The bolometric flux of the unabsorbed best-fit model spectrum.

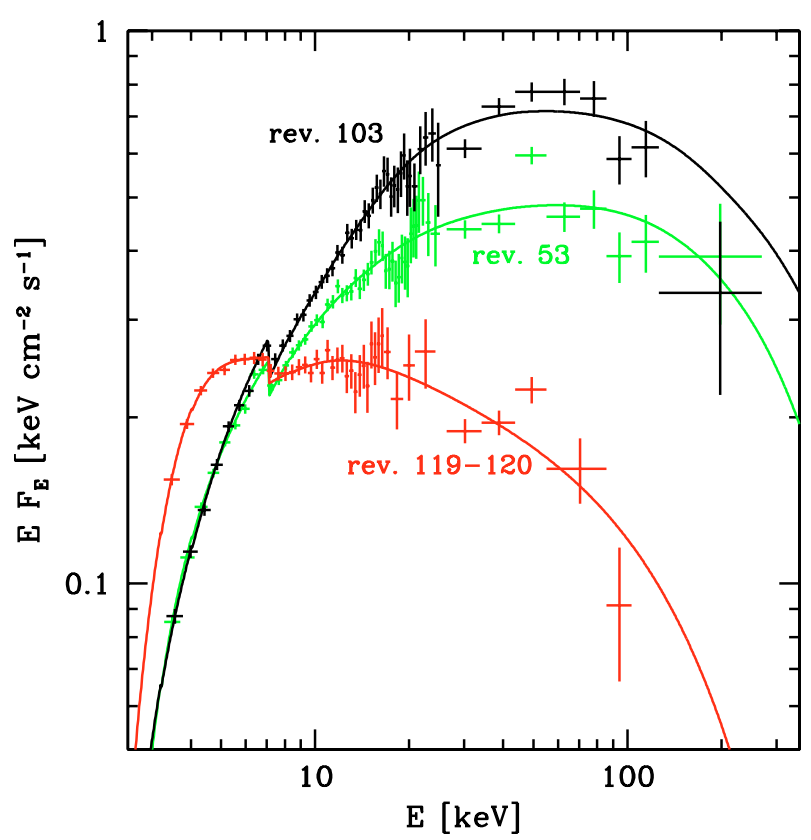

Fig. 2. The observed (absorbed) spectra in rev. 53 (hard state), rev. 103 (hard state) and rev. 119-120 (soft state), as fitted by the thermal Comptonization model COMPPS.

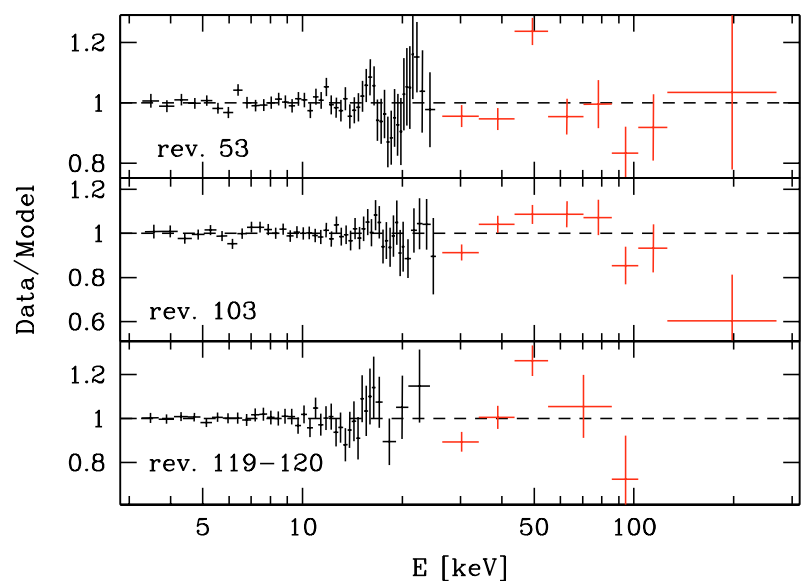

Fig. 3. Residuals for the three data sets of PCA (black, $E<25 \mathrm{keV}$ ) and IBIS (red, $E>25 \mathrm{keV}$ ).

weakest in the last data set, which is not typical of black-hole binaries (Gierliński et al. 1999).

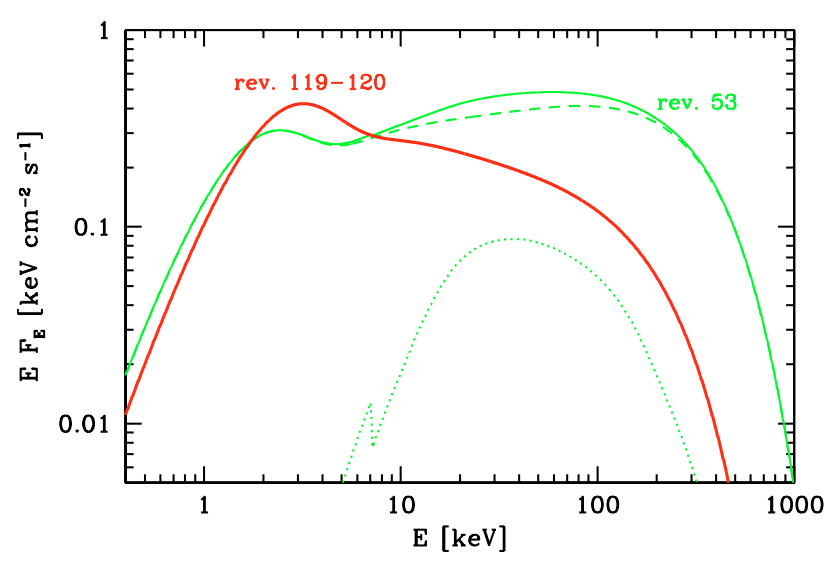

Fig. 4. The unabsorbed thermal Comptonization for rev. 53 (green) and 119-120 (red). For the first spectrum, its components due to thermal Comptonization of blackbody photons (dashed curve) and Compton reflection (dotted curve) are shown.

\section{Discussion and conclusions}

We have observed several variability patterns of 1E 1740.7-2942. First the estimated bolometric flux increased by $\sim 1.5$ in 6 months (2003 February-August) with an almost constant spectral shape. The spectrum, with a hard $\mathrm{X}$-ray index, a Compton-reflection component, and a cutoff corresponding to $k T_{\mathrm{e}} \sim 100 \mathrm{keV}$, is typical of the low/hard state of black-hole binaries, e.g., Cyg X-1 (Gierliński et al. 1997) or GX 339-4 (Wardziński et al. 2002).

However, we observed a spectral state transition. In 2003 October, the flux in the low-energy part of the spectrum increased, and our fits show an increase of the disc blackbody temperature from $0.4-0.5 \mathrm{keV}$ in the hard state up to $\sim 0.7 \mathrm{keV}$. The power law had substantially softened, with $\Gamma$ increasing from 1.3-1.5 to $\sim 2.3$. These spectral characteristics are peculiar to the soft state, e.g., of Cyg X-1 (Gierliński et al. 1999).

Interestingly, the flux of this state was significantly lower than that of the hard state. Although the opposite behaviour, with the flux in the soft state larger than that in the hard state, is most typical of black-hole binaries, the behaviour observed by us is also seen in other low-mass X-ray binaries, e.g., in GX 339-4. Smith et al. (2002a) often observed GRS 1758-258 at low luminosities in the typical soft state. This is due to the existence of two accretion solutions at a given accretion rate, which allows for such a hysteretic behaviour (e.g., Zdziarski et al. 2004). This transient-like type of behaviour is 
consistent with occasional quenching of the X-ray emission of 1E 1740.7-2942, as confirmed by our study.

Assuming a distance of $8.5 \mathrm{kpc}$ and $10 M_{\odot}$ for the compact object, we estimated the bolometric luminosity of 1E 1740.7-2942 during rev. 53, rev. 103 and rev. 119-120 to be $\sim 0.01 L_{\text {Edd }}, \sim 0.03 L_{\text {Edd }}$ and $\sim 0.02 L_{\text {Edd }}$, respectively. Our results are consistent with Maccarone (2003), who shows that the state transition luminosities are at about $1-4 \%$ of the Eddington rate for most of the X-ray binaries.

An interesting feature of the observed soft state is the unusual weakness of the Compton reflection component, which usually becomes stronger in the soft state (e.g., Gierliński et al. 1999) than in the hard state. We suggest that it is possible that the accretion disc in 1E 1740.7-2942 precesses, and we could have caught the source at the moment when the disc was edge-on, causing the reflection to be not observable.

In our spectra integrated over time scales of $20 \mathrm{ks}$ and $100 \mathrm{ks}$, there is no evidence of any high energy feature. In the energy range $485-535 \mathrm{keV}$ in $\sim 1.4 \mathrm{Ms}$ integration time, De Cesare et al. (2004) report an upper limit at the $2 \sigma$ level of roughly $2.0 \times 10^{-4} \mathrm{ph} \mathrm{cm}^{-2} \mathrm{~s}^{-1}$, including systematic errors. Based on this result, the $2 \sigma$ upper limits for our time scales of $20 \mathrm{ks}$ and $100 \mathrm{ks}$ are $1.7 \times 10^{-3}$ and $7.4 \times 10^{-4} \mathrm{ph} \mathrm{cm}^{-2} \mathrm{~s}^{-1}$, respectively.

Acknowledgements. This work has been supported by ASI via contract I/R/389/02 and I/R/041/02. MDS thanks Julien Malzac for precious scientific suggestions, Memmo Federici for the data archive support and Angelique Joinet for useful data analysis discussion. A.A.Z. has been supported by KBN grants PBZ-KBN-054/P03/2001 and 1P03D01827. We thank A. Parmar for providing access to proprietaries data.

\section{References}

Bouchet, L., Mandrou, P., Roques, J. P., et al. 1991, ApJ, 383, L45 Cadolle Bel, M., Rodriguez, J., Sizun, P., et al. 2004, A\&A, in press [arXiv: astro-ph/0407308]

Cordier, B., Paul, J., Ballet, J., et al. 1993, A\&A, 275, L1

Cordier, B., Paul, J., \& Hameury, J.-M. 1994, ApJS, 92, 401

Courvoisier, T. J.-L., Walter, R., Beckmann, V., et al. 2003, A\&A, 411, L53

De Cesare, G., Bazzano, A., Capitanio, F., et al. 2004, COSPAR 2004 proceedings, submitted
Del Santo, M., Bazzano, A., Smith, D. M., et al. 2004, Proc. of the 5th INTEGRAL Workshop, ESA SP552

Gallo, E., \& Fender, R. P. 2002, MNRAS, 337, 869

Gierliński, M., Zdziarski, A. A., Done, C., et al. 1997, MNRAS, 288, 958

Gierliński, M., Zdziarski, A. A., Poutanen, J., et al. 1999, MNRAS, 309,496

Goldwurm, A., David, P., Foschini, L., et al. 2003, A\&A, 411, L223

Grebenev, S. A., Revnivtsev, M. G., \& Sunyaev, R. A. 2004, ATel, 257

Hertz, P., \& Grindlay, J. E. 1984, ApJ, 278, 137

Jung, G. V., Kurfess, D. J., Johnson, W. N., et al. 1995, A\&A, 295, L23

Lebrun, F., Leray, J. P., Lavocat, P., et al. 2003, A\&A, 411, L141

Maccarone, T. J. 2003, A\&A, 409, 697

Magdziarz, P., \& Zdziarski, A. A. 1995, MNRAS, 273, 837

Main, D. S., Smith, D. M., Heindl, W. A., et al. 1999, ApJ, 525, 901

Martí, J., Mirabel, I. F., Chaty, S., \& Rodríguez, L. F. 2000, A\&A, 363,184

Mirabel, I. F., Rodriguez, L. F., Cordier, B., Paul, J., \& Lebrun, F. 1992, Nature, 358, 215

Parmar, A. N., Kuulkers, E., Oosterbroek, T., et al. 2003, A\&A, 411, L421

Poutanen, J., \& Svensson, R. 1996, ApJ, 470, 249

Revnivtsev, M., Chernyakova, M., Capitanio, F., et al. 2003, ATel, 132

Sakano, M., Imanishi, K., Tsujimoto, M., Koyama, K., \& Maeda, Y. 1999, ApJ, 520, 316

Sidoli, L., Mereghetti, S., Israel, G. L., et al. 1999, ApJ, 525, 215

Skinner, G. K., Willmore, A. P., Eyles, C. J., Bertram, D., \& Church, M. J. 1987, Nature, 330, 544

Smith, D. M., Heindl, W. A., \& Swank, J. H. 2002a, ApJ, 569, 362

Smith, D. M., Heindl, W. A., \& Swank, J. H. 2002b, ApJ, 578, L129

Sunyaev, R., Churazov, E., Gilfanov, M., et al. 1991a, A\&A, 247, L29

Sunyaev, R., Churazov, E., Gilfanov, M., et al. 1991b, ApJ, 383, L49

Titarchuk, L. 1994, ApJ, 434, 313

Ubertini, P., Lebrun, F., Di Cocco, G., et al. 2003, A\&A, 411, L131

Wardziński, G., Zdziarski, A. A., Gierliński, M., et al. 2002, MNRAS, 337,829

Winkler, C., Gehrels, N., Lund, N., et al. 1999, Astron. Lett. Comm., 39,361

Zdziarski, A. A., \& Gierliński, M. 2004, Prog. Theor. Phys. Suppl., 155,99

Zdziarski, A. A., Johnson, W. N., \& Magdziarz, P. 1996, MNRAS, 283, 193

Zdziarski, A. A., Poutanen, J., \& Johnson, W. N. 2000, ApJ, 542, 703

Zdziarski, A. A., Lubiński, P., Gilfanov, M., \& Revnivtsev, M. 2003, MNRAS, 342, 355

Zdziarski, A. A., Gierliński, M., Mikołajewska, J., et al. 2004, MNRAS, 351, 791 